\title{
Incidence of self reported glaucoma in people with diabetes mellitus
}

\author{
Barbara E K Klein, Ronald Klein, Scot E Moss
}

\begin{abstract}
Aim-To determine the incidence of self reported glaucoma in a group of people with diabetes mellitus.

Methods-In an 11 county area in southern Wisconsin, a cohort of people with diabetes mellitus was identified and characterised by history, physical examination, fundus photographs, and laboratory evaluation in 1980-2 $(n=2366)$. The cohort was followed for 10 years with 891 younger onset and 987 older onset people participating in at least one follow up examination.

Results-The 10 year incidence of glaucoma was $3.7 \%$ in younger onset people, $6.9 \%$ in older onset people not using insulin, and $11.8 \%$ in older onset people using insulin. Univariate analyses revealed that in younger onset people greater body mass index, older age, longer duration of diabetes, history of cataract surgery, and increased severity of diabetic retinopathy at baseline conferred increased risk. In older onset people using insulin, older age and longer duration of diabetes, higher intraocular pressure, and history of cataract surgery at baseline were associated with increased risk of glaucoma. In multivariate analyses, only age was significantly related to increased incidence in younger onset people and age, intraocular pressure, and insulin use were significantly associated with increased risk in the older onset group.
\end{abstract}

Conclusion-Most risk factors associated with self reported glaucoma in people with diabetes cannot currently be modified to decrease incidence. Older onset people who are at increased risk of many ocular complications of diabetes may be particularly in need of specialised ophthalmic observation.

(Br F Ophthalmol 1997;81:743-747)

Diabetes mellitus confers several risks to the eye including retinopathy, ${ }^{1-4}$ certain kinds of lens opacities (cataract), ${ }^{5}$ increased intraocular pressure, ${ }^{6}$ rubeosis iridis, ${ }^{6}$ and possibly open angle glaucoma. ${ }^{6}$ Many of the studies of glaucoma in people with diabetes derive from clinic populations and are not prospective in design. There is a dearth of information concerning the incidence of glaucoma and factors that affect it in people with diabetes. We had an opportunity to examine incidence of glaucoma by self report in a cohort study of people with diabetes who have been followed for a 10 year period.

\section{Materials and methods}

The Wisconsin Epidemiologic Study of Diabetic Retinopathy (WESDR) is a population based study of the prevalence and incidence of diabetic retinopathy. The methods used in identifying the population, and results describing the study findings, are reported in previous publications $s^{1-48}$ and are summarised here. The State Division of Health provided a list of all of the 500 primary care physicians who were practising medicine in July 1979 in an 11 county area in southern Wisconsin (population 839 324). ${ }^{2}$ Primary care physicians included general practitioners $(n=190)$, family practitioners $(n=96)$, internists $(n=155)$, and paediatricians $(n=59)$ and included physicians practising in diabetes specialty clinics. Physicians in residency or fellowship training programmes or who were practising specialties not associated with primary care for diabetes, such as ophthalmology, radiology, pathology, anaesthesiology, and psychiatry, were excluded. Physicians received a telephone call followed by a letter describing the objectives and design of the proposed study and inviting them and their diabetic patients to participate in the study. Of the 500 primary care practitioners, 452 $(90.4 \%)$ chose to participate. Forty three of the 48 non-participating physicians stated that they did not provide primary care for diabetic patients; five (three internists, one general practitioner, and one family practitioner) refused to participate.

Two methods of identifying patients were used-daily lists with names entered by hand by the physician or his/her staff, and computer retrieval of records of patients with a coded diagnosis of diabetes. Over the 1 year period ( 1 July 1979 to 30 June 1980), study workers visited all the medical offices and established record keeping procedures. Handwritten lists of patients were kept for 142 offices, involving 288 physicians; computer retrieval was used in 18 offices involving 164 physicians.

In this manner, 10135 patients were identified and patient charts were reviewed for 9841 of these. Failure to review charts was due to inability to find the charts during this period $(n=177)$, and patient refusal to allow chart review $(n=117)$. Of the 9841 patients whose charts were reviewed, 338 patients were confined to a nursing home, 157 had died before 1 July 1979, 45 did not have diabetes (incorrect computer coding), and 18 either had moved before 1 July 1979 or had gestational 
diabetes. The charts of these 558 patients were reviewed for sociodemographic data only.

Review of the remaining 9283 charts was done according to a written protocol by a team consisting of a physician and two registered nurses. The information so abstracted included patient's date of birth, sex, date of diagnosis of diabetes, blood sugars, and type of hypoglycaemic medication, if any, currently being used. The criteria used for inclusion of cases of diabetes were that the patients: (1) had been diagnosed as having diabetes by a physician; (2) were considered to be under the primary care of the participating physician during the period 1 July 1979 to 30 June 1980 ; (3) were alive and living in the 11 county area during the same period. There were 8135 cases who met these criteria.

A sample of 2990 subjects was selected for the baseline examination. The sample was composed of two groups: the first group included all 1210 subjects who were diagnosed to have diabetes before age 30 and who took insulin (younger onset) ${ }^{1}$; the second group consisted of a sample of 1780 subjects diagnosed with diabetes at age 30 or older (older onset). ${ }^{3}$ This latter group consisted of a probability sample of older onset subjects, stratified by duration of diabetes in order to have an adequate number of subjects in each duration group to perform meaningful statistical analyses. Therefore, there were 576 people with duration of diabetes of less than 5 years, 579 people with $5-14$ years' duration, and 625 people with greater than 15 years' duration who were selected for inclusion in the study.

Institutionalised people, such as nursing home residents, who were unable to leave the institution for care of their diabetes, were not included. Tenets of the Declaration of Helsinki were followed. Informed consent was signed and institutional human experimentation committee approval was granted.

Of the younger onset people, 996 participated in the baseline examination (1980 to 1982), ${ }^{1} 891$ in the 4 year follow up, ${ }^{2}$ and 765 in the 10 year follow up. ${ }^{8}$ Of the 1780 eligible older onset people, 1370 participated in the baseline examination, ${ }^{3} 987$ in the 4 year follow up, ${ }^{4}$ and 533 in the 10 year follow up. ${ }^{8}$ Reasons for non-participation and comparisons between participants and non-participants at baseline and the 4 year follow up have been presented elsewhere. ${ }^{1-4}$ The mean time between the baseline and the follow up examinations was 10.1 years (SD 0.4 years).

Blood pressure was measured according to the Hypertension Detection and Follow-up Program protocol. ${ }^{9}$ Weight and height were measured by protocol. The body mass index was weight $(\mathrm{kg})$ divided by height $(\mathrm{m})$ squared. Each subject's eyes were refracted and the distance visual acuity measured and recorded using the $\log$ MAR chart, following the Early Treatment Diabetic Retinopathy Study protocol. ${ }^{10}$ The anterior chamber was examined at the slit lamp for evidence of new vessels on the iris as judged by protocol with reference to a photographic standard and for anterior chamber depth to assess the possibility of angle closure after pupillary dilatation. The slit lamp examination was designed for the needs of this study by the investigators. The photographic standard was selected specifically for identification of new vessels on the iris. ${ }^{6}$ Intraocular pressure was measured using a Goldmann applanation tonometer according to standardised protocol. ${ }^{6}$ Duration of diabetes was the time between diagnosis and the baseline examination. During each study interview, subjects were queried as to whether or not they took medication for diabetes and, if so, the names and doses of such preparations. In addition, they were asked whether they were taking medicine for glaucoma. Subjects who responded that they took medication for glaucoma and those with slit lamp evidence of new vessels on the iris (rubeosis iridis) at the first examination were excluded from consideration as incident cases of glaucoma. Those who were not cases at the baseline but who responded affirmatively to the same question concerning use of medication for glaucoma at the follow up were considered incident cases. Those with new evidence of rubeosis at follow up were excluded from incidence calculations, thus eliminating cases of neovascular glaucoma. There were no cases with evidence of narrow angle glaucoma or other secondary causes of glaucoma.

When evaluating risk factors, responses to questions at the baseline examination were used in the analyses. Individuals were asked whether they had smoked more than 100 cigarettes in their lives and, if so, how many cigarettes per day they smoked and for how many years (one pack year was equivalent to smoking one pack per day for each year); and if a doctor ever told them that they had had a heart attack, angina, or a stroke. Cataract surgery was based on the lens examination.

Colour stereoscopic fundus photographs were taken of each eye. They were subsequently graded and classified according to the Modified Airlie House Classification as described by the Early Treatment Diabetic Retinopathy Study. ${ }^{211}$ In general, gradings are ranked as to severity. The scheme specifies 13 levels of severity of retinopathy. For the analyses presented, the level in the worse eye was used and was grouped into four categories: no retinopathy (level 10), mild non-proliferative retinopathy (levels 21 to 37), moderate nonproliferative retinopathy (levels 43 to 53), and proliferative diabetic retinopathy (PDR) (levels $60+)$.

Glycosylated haemoglobin was measured by a microcolumn technique on a specimen of capillary blood. ${ }^{12}$ Gross proteinuria was defined as a urine concentration of $0.30 \mathrm{~g} / \mathrm{l}$ or more measured by a reagent strip. Quartile values of glycosylated haemoglobin and blood pressure were determined from the distribution of values for younger and older onset people from data at the first examination.

Because some participants observed at the 1984-6 examination did not return for the 1990-2 examination (censored observations), the product limit method was used to calculate 10 year rates of glaucoma. ${ }^{13}$ This procedure has 


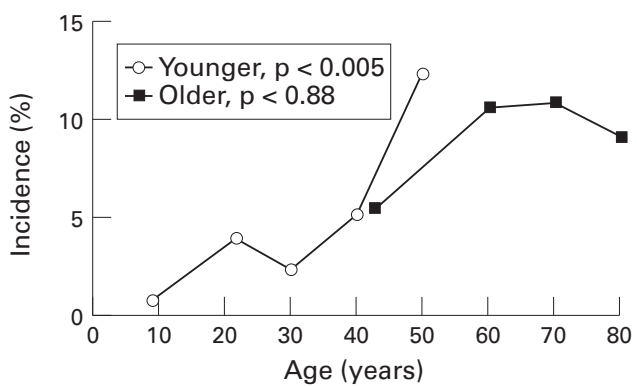

Figure 1 Ten year incidence of history of glaucoma by age for younger and older onset diabetic people in the Wisconsin Epidemiologic Study of Diabetic Retinopathy.

the advantage of using all the information from each follow up period. To calculate relative risks and test for trends, the methods of Mantel and Haenszel, stratified by the follow up period, were used. ${ }^{14}$ Multivariate analyses were performed with the discrete linear logistic model. ${ }^{15}$

\section{Results}

Baseline values for relevant characteristics for the younger onset group were as follows: (mean (SD)) age 28.3 (12.4) years, duration of diabetes 13.8 (9.8) years, glycosylated haemoglobin $10.8 \%(2.1 \%)$, systolic blood pressure 123 (19) $\mathrm{mm} \mathrm{Hg}$, diastolic blood pressure 79 (11) $\mathrm{mm} \mathrm{Hg}$, and insulin dose $0.75(0.32)$ units $/ \mathrm{kg} /$ day. Values for the older onset group were: mean age 64.6 (11.0) years, duration of diabetes 11.2 (8.0) years, glycosylated haemoglobin 9.6 (2.0), systolic blood pressure 146 (23) $\mathrm{mm} \mathrm{Hg}$, and diastolic blood pressure 80 (12) $\mathrm{mm} \mathrm{Hg}$. Of the younger onset group, $50.5 \%$ were men and $1.1 \%$ had a history of glaucoma at baseline. Of the older onset group, $45.1 \%$ were men, $49.1 \%$ took insulin, and $3.4 \%$ had glaucoma. The incidence of glaucoma over the 10 year period was 3.7\% (95\% confidence interval (CI) $2.3,5.1$ ) in younger onset people, $6.9 \%(95 \%$ CI $3.9,9.8)$ in older onset people not using insulin, and 11.8 (95\% CI $7.9,15.7$ ) in those taking insulin.

Incidence of glaucoma varied with age (Fig 1 ). In younger onset people and in older onset people (combining both users and non-users of insulin), incidence increases with age, although only in the former group did the relation reach conventional levels of statistical significance.

The relation of duration of diabetes to glaucoma is seen in Figure 2. The relation is significant in both groups.

Table 1 describes the univariate relation between other baseline characteristics and incidence of glaucoma in both groups. Cataract surgery at baseline is significantly associated with incident glaucoma in both groups. Body mass index and severity of diabetic retinopathy are associated with risk in younger onset people, while intraocular pressure and insulin use are associated in older onset people.

In order to evaluate the effects of several factors on the incidence of glaucoma simultaneously, discrete linear logistic regression analyses are employed (Table 2). The variables considered are age, sex, glycosylated haemoglobin, duration of diabetes, systolic and

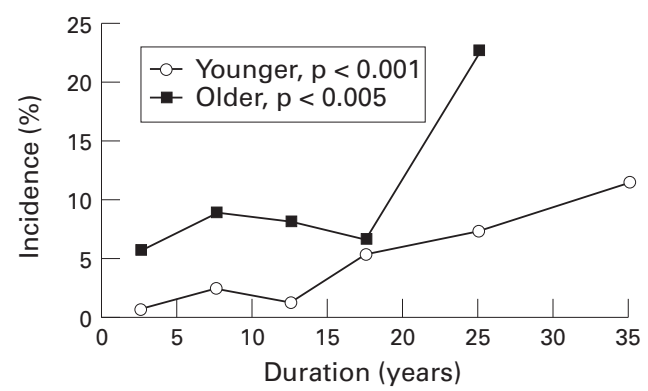

Figure 2 Ten year incidence of history of glaucoma by duration of diabetes for younger and older onset diabetic people in the Wisconsin Epidemiologic Study of Diabetic people in the
Retinopathy.

diastolic blood pressure, body mass index, pack years smoked, proteinuria, intraocular pressure, cataract surgery, and use of insulin in older onset people. In younger onset people only age is significantly related to incidence. In older onset people age, intraocular pressure, and insulin use are significantly associated with increased risk. Cataract surgery is of borderline significance.

\section{Discussion}

The people participating in the WESDR are a population based cohort. We had a high participation rate among area physicians and it seems unlikely that reasons of nonparticipation of physicians is likely to affect analyses here. Sampling probabilities are known and estimates derived from these data would be expected to be representative of similar population based studies of complications of diabetes in similar populations.

While non-participants at every examination in the WESDR had, on average, longer duration of diabetes, were older, and had higher blood pressures, we are unable to estimate the effects of these variables on the relation we report. This may have caused us to underestimate effects of blood pressure, glycosylated haemoglobin, cardiovascular disease, proteinuria, sex, and retinopathy as these characteristics are themselves risk factors for mortality and other significant morbidities. We are unable to address the relation in people sequestered in nursing homes or other institutional settings because of the design of this study. As with other non-participants, omission of these people may have caused an underestimate of risk associated with the systemic factors just listed. In addition, these people may have had higher rates of glaucoma causing disability serious enough that, in combination with other frailties, were the reason for nursing home placement. These considerations are less likely to be worrisome in data from the younger onset group in which non-participation has not been a major concern.

Diabetes confers risk of retinopathy ${ }^{1-4}$ and increased risk of lens opacity. ${ }^{5}$ In the same population participating in this study, we reported a higher prevalence of history of glaucoma in both diabetic groups compared with participants in the Health Interview Survey and in a non-diabetic comparison group. ${ }^{6}$ In the Beaver Dam Eye Study, people with 
Table 1 Ten year incidence of glaucoma in younger and older onset diabetic people by baseline characteristics in the Wisconsin Epidemiologic Study of Diabetic Retinopathy (1979-92)

\begin{tabular}{|c|c|c|c|c|c|c|c|c|}
\hline \multirow[b]{3}{*}{ Characteristic } & \multicolumn{4}{|c|}{ Younger onset } & \multicolumn{4}{|c|}{ Older onset } \\
\hline & \multicolumn{2}{|c|}{ Incidence } & \multirow[b]{2}{*}{$95 \% C I$} & \multirow[b]{2}{*}{$p^{*}$} & \multicolumn{2}{|c|}{ Incidence } & \multirow[b]{2}{*}{$95 \% C I$} & \multirow[b]{2}{*}{$p^{\star}$} \\
\hline & $N$ & $(\%)$ & & & $N$ & $(\%)$ & & \\
\hline \multicolumn{9}{|l|}{ Blood pressure: } \\
\hline \multicolumn{9}{|l|}{ Systolic } \\
\hline Quartile 1 & 199 & 3.2 & $0.7,5.8$ & \multirow[t]{4}{*}{0.47} & 226 & 6.9 & $2.8,10.9$ & \multirow[t]{4}{*}{0.20} \\
\hline Quartile 2 & 220 & 4.0 & $1.3,6.7$ & & 240 & 8.4 & $4.2,12.5$ & \\
\hline Quartile 3 & 197 & 1.7 & $0.0,3.6$ & & 223 & 13.4 & $7.8,19.1$ & \\
\hline Quartile 4 & 167 & 6.0 & $1.9,10.0$ & & 198 & 7.9 & $2.2,13.6$ & \\
\hline \multicolumn{9}{|l|}{ Diastolic } \\
\hline Quartile 1 & 210 & 3.1 & $0.7,5.5$ & \multirow[t]{4}{*}{0.37} & 197 & 7.0 & $2.4,11.5$ & \multirow[t]{4}{*}{0.77} \\
\hline Quartile 2 & 199 & 6.4 & $2.9,9.9$ & & 241 & 8.6 & $4.3,13.0$ & \\
\hline Quartile 3 & 187 & 1.9 & $0.0,4.0$ & & 236 & 11.9 & $6.5,17.3$ & \\
\hline Quartile 4 & 186 & 2.8 & $0.1,5.4$ & & 210 & 8.6 & $3.9,13.2$ & \\
\hline \multicolumn{9}{|l|}{ BMI: } \\
\hline Quartile 1 & 208 & 1.5 & $0.0,3.2$ & 0.05 & 193 & 8.2 & $3.5,12.9$ & 0.95 \\
\hline Quartile 2 & 195 & 4.6 & $1.5,7.7$ & & 226 & 8.0 & $3.6,12.5$ & \\
\hline Quartile 3 & 197 & 2.4 & $0.1,4.7$ & & 230 & 11.3 & $5.8,16.9$ & \\
\hline Quartile 4 & 188 & 6.5 & $2.8,10.3$ & & 239 & 9.0 & $4.6,13.3$ & \\
\hline $\mathrm{HbA}_{1}:$ & & & & & & & & \\
\hline Quartile 1 & 198 & 2.8 & $0.4,5.2$ & 0.50 & 225 & 10.2 & $5.6,14.8$ & 0.55 \\
\hline Quartile 2 & 183 & 6.3 & $2.5,10.1$ & & 203 & 6.6 & $2.0,11.2$ & \\
\hline Quartile 3 & 201 & 4.0 & $1.1,6.9$ & & 204 & 7.2 & $2.7,11.8$ & \\
\hline Quartile 4 & 173 & 1.9 & $0.0,4.0$ & & 190 & 12.8 & $6.4,19.1$ & \\
\hline History of cardi & & & & & & & & \\
\hline - & 768 & 3.6 & $2.2,5.0$ & 0.60 & 676 & 9.5 & $6.8,12.2$ & 0.32 \\
\hline+ & 21 & 6.7 & $0.0,19.3$ & & 209 & 7.3 & $1.9,12.7$ & \\
\hline Insulin: & & & & & & & & \\
\hline Not using & - & - & - & & 458 & 6.9 & $3.9,9.8$ & 0.05 \\
\hline Using & - & - & - & & 430 & 11.8 & $7.9,15.7$ & \\
\hline Proteinuria: & & & & & & & & \\
\hline Absent & 625 & 3.4 & $1.9,4.8$ & 0.28 & 761 & 8.7 & $6.2,11.1$ & 0.52 \\
\hline Present & 133 & 5.3 & $1.2,9.5$ & & 98 & 10.7 & $1.1,20.3$ & \\
\hline Sex: & & & & & & & & \\
\hline Female & 387 & 4.5 & $2.3,6.7$ & 0.24 & 485 & 9.0 & $6.0,12.1$ & 0.65 \\
\hline Male & 402 & 2.9 & $1.1,4.6$ & & 403 & 9.5 & $5.6,13.4$ & \\
\hline Cataract surgery & & & & & & & & \\
\hline - & 751 & 3.1 & $1.8,4.4$ & $<0.05$ & 819 & 8.2 & $5.8,10.6$ & $<0.005$ \\
\hline+ & 16 & 14.1 & $0.0,32.3$ & & 49 & 27.0 & $8.2,45.8$ & \\
\hline Intraocular pres & & & & & & & & \\
\hline$<14$ & 198 & 2.8 & $0.4,5.3$ & 0.17 & 208 & 3.3 & $0.3,6.4$ & $<0.001$ \\
\hline $14-15$ & 174 & 3.8 & $0.8,6.7$ & & 182 & 5.1 & $0.9,9.3$ & \\
\hline $16-18$ & 230 & 1.9 & $0.1,3.8$ & & 254 & 7.9 & $3.9,11.9$ & \\
\hline$>18$ & 165 & 6.9 & $2.8,11.0$ & & 226 & 18.3 & $11.9,24.7$ & \\
\hline Refractive error & & & & & & & & \\
\hline$\leqslant-3$ & 130 & 3.4 & $0.1,6.7$ & 0.84 & 48 & 23.5 & $9.1,37.9$ & 0.08 \\
\hline$>-3, \leqslant-1$ & 201 & 3.9 & $1.1,6.8$ & & 64 & 12.1 & $2.5,21.7$ & \\
\hline$>-1,<1$ & 407 & 3.3 & $1.5,5.1$ & & 373 & 8.6 & $5.1,12.2$ & \\
\hline$\geqslant 1,<3$ & $37 \dagger$ & $6.1 \dagger$ & $0.0,14.3$ & & 286 & 5.1 & $1.8,8.4$ & \\
\hline$\geqslant 3$ & - & - & - & & 104 & 11.8 & $4.0,19.6$ & \\
\hline Retinopathy: & & & & & & & & \\
\hline None & 238 & 1.8 & $0.0,3.6$ & $<0.05$ & 410 & 10.0 & $6.5,13.6$ & 0.68 \\
\hline Mild & 323 & 3.7 & $1.6,5.9$ & & 328 & 7.1 & $3.6,10.6$ & \\
\hline Moderate & 89 & 3.8 & $0.0,8.1$ & & 93 & 11.5 & $1.7,21.3$ & \\
\hline PDR & 139 & 7.2 & $2.3,12.1$ & & 57 & 8.8 & $1.4,16.1$ & \\
\hline
\end{tabular}

$\mathrm{CI}=$ confidence interval; $\mathrm{HbA}_{1}=$ glycosylated haemoglobin; $\mathrm{BMI}=$ body mass index

$\star$ Test of trend for characteristics with more than two categories.

†For younger onset, number and rate for $\geqslant 1$.

diabetes had an increased prevalence of glaucoma. ${ }^{16}$ This was true for those who met the codified examination criteria (definite glaucoma) and those with history of glaucoma defined in a similar manner to that used in the current paper (probable glaucoma), although in the latter group the relative odds (1.47) did not meet a level of statistical significance

Table 2 Characteristics associated with incidence of glaucoma in multivariate analysis by discrete linear logistic models in the Wisconsin Epidemiologic Study of Diabetic Retinopathy (1979-92)

\begin{tabular}{lllll}
\hline & $\begin{array}{l}\text { Unit } \\
\text { difference }\end{array}$ & $p$ Value & OR & $95 \% C I$ \\
\hline $\begin{array}{lllll}\text { Younger onset: } \\
\quad \text { Age }\end{array}$ & 10 years & $<0.0001$ & 1.77 & $1.33,2.35$ \\
$\begin{array}{l}\text { Older onset: } \\
\text { Age }\end{array}$ & 10 years & $<0.05$ & 1.37 & $1.01,1.86$ \\
IOP & 1 mm Hg & $<0.0001$ & 1.25 & $1.16,1.35$ \\
Insulin & Taking & $<0.005$ & 2.66 & $1.43,4.94$ \\
Cataract surgery & Yes & 0.06 & 2.39 & $0.98,5.86$ \\
\hline
\end{tabular}

$(p=0.18) .{ }^{16}{ }^{17}$ There is a paucity of incidence data to compare with our findings. Thus, we can only state that the incidence is substantial and appears to be considerably higher than prevalence findings in general populations.

As in non-diabetic populations, increasing age confers increasing risk. Because of the effects of age on mortality in the intervals between examinations, the burden of glaucoma in all groups may be underestimated in our analyses. On the other hand, there is concern when using historical information for diagnosis of glaucoma that doctors treat intraocular pressures in people with diabetes prophylactically to decrease the risk of optic nerve and visual field changes. Thus, people classified as having disease based on medication usage may not have evidence of pathology. However, data from the older onset group indicate a monotonic increase in risk with increasing intraocular 
pressure at baseline 10 years before the follow up, beginning at relatively low levels of pressure (Table 1). The importance of intraocular pressure as a risk factor for glaucoma has been confirmed in many studies ${ }^{18-21}$ and its effect may be enhanced by the presence of diabetes. ${ }^{22}$ A trend, although not nearly as convincing nor significant, is apparent in the younger onset group. It may be that an intraocular pressure 'effect' will become more apparent in the younger onset group as it achieves the age range of the older onset group.

Cataract surgery is associated with increased incidence in both groups. It is not possible from these data to determine whether the association is related to a causal pathophysiological effect of cataract or cataract surgery on incident glaucoma or is a result of greater medical scrutiny in those who have undergone a surgical procedure.

Increased severity of diabetic retinopathy is associated in univariate analyses with subsequent incident glaucoma. This factor is not significant when age is accounted for in the logistic model. Whether this is for biological reasons or a result of covariance cannot be distinguished in these analyses.

We make the assumption that the large majority of cases of glaucoma, as we define it, are open angle glaucoma because no cases of narrow angle glaucoma, or secondary causes except for iris neovascularisation, were noted. We cannot be certain that other causes of glaucoma have been entirely excluded, but would expect such cases to be rare or non-existent in our small number of cases.

The usual concern is that glaucoma is under-reported and underdiagnosed in a general population. People with diabetes are under medical observation for their diabetes and a large proportion of these patients are under the care of an ophthalmologist. ${ }^{23}$ However, it is possible still that we may have missed some cases of this disease.

Stereoscopic photographs of the optic discs of all study subjects were taken at the baseline and graded according to codified procedures. These prevalence data indicated larger cup to disc ratios in people with glaucoma in the older onset but not younger onset group. Thus, although not a test of the validity of self report, the gradings were consistent with the historical data. ${ }^{24}$

It appears that the risk of glaucoma is greatest in those people with older onset diabetes who are taking insulin. This group has also been shown to be at increased risk of other ocular complications of diabetes. Thus, older onset people represent a group who may be particularly in need of specialised ophthalmic observation.

This research is supported by NIH grant EYO3083 and, in part, by the Research to Prevent Blindness (R Klein, senior scientific investigator award).

1 Klein R, Klein BEK, Moss SE, Davis MD, DeMets DL. The Wisconsin Epidemiologic Study of Diabetic Retinopathy. II Prevalence and risk of diabetic retinopathy when age at diagnosis is less than 30 years. Arch Ophthalmol 1984;102:520-6

2 Klein R, Klein BEK, Moss SE, Davis MD, DeMets DL. The Wisconsin Epidemiologic Study of Diabetic Retinopathy. IX Four-year incidence and progression of diabetic retinopathy when age at diagnosis is less than 30 years. Arch Ophthalmol 1989;107:237-43.

3 Klein R, Klein BEK, Moss SE, Davis MD, DeMets DL. The Wisconsin Epidemiologic Study of Diabetic Retinopathy. III Prevalence and risk of diabetic retinopathy when age at diagnosis is 30 or more years. Arch Ophthalmol 1984;102: diagnosis

4 Klein R, Klein BEK, Moss SE, Davis MD, DeMets DL. The Wisconsin Epidemiologic Study of Diabetic Retinopathy. $\mathrm{X}$ Four-year incidence and progression of diabetic retinopathy when age at diagnosis is 30 years or more. Arch Ophthalmol 1989;107:244-9.

5 Klein BEK, Klein R, Wang Q, Moss SE. Older-onset diabetes and lens opacities. The Beaver Dam Eye Study. Ophthalmic Epidemiol 1995;2:49-55.

6 Klein BEK, Klein R, Moss SE. Intraocular pressure in diabetic persons. Ophthalmology 1984;91:1356-60.

7 Klein R, Klein BEK, Moss SE, DeMets DL, Kaufman I, Voss PS. Prevalence of diabetes mellitus in southern Wisconsin. Am f Epidemiol 1984;119:54-61.

8 Klein R, Klein BEK, Moss SE, Cruickshanks KJ. The Wisconsin Epidemiologic Study of Diabetic Retinopathy. XIV Ten-year incidence and progression of diabetic retinopathy. Ten-year incidence and progression

9 Hypertension Detection and Follow-up Program Cooperative Group. The hypertension detection and follow-up protive Group. The hypertension deted
gram. Prev Med 1976;5:207-15.

gram. Prev Med 1976;5:207-15.
10 Early Treatment Diabetic Retinopathy Study Coordinating Center. Manual of operations. Baltimore: Diabetic Retinopathy Study Coordinating Center, 1985; Chapter 13. Available from National Technical Information Service (Accession No PB 85-223006/AS).

11 Klein BEK, Davis MD, Segal P, Long JA, Harris WA, Haug GA, et al. Diabetic retinopathy: assessment of severity and progression. Ophthalmology 1984;91:10-7.

12 Quick-Step. Fast Hemoglobin Test System. Akron, OH: Isolab, 1981.

13 Kaplan EL, Meier P. Nonparametric estimation from incomplete observations. $\mathcal{F}$ Am Stat Assoc 1958;53:457-81.

14 Mantel N. Chi-square tests with one degree of freedom: extensions of the Mantel-Haenszel procedure. $7 \mathrm{Am}$ Stat extensions of the Mante-

15 Hosmer DW Jr, Lemeshow S. Applied logistic regression. New York: Wiley, 1989:238-45.

16 Klein BEK, Klein R, Jensen SC. Open-angle glaucoma and older-onset diabetes. The Beaver Dam Eye Study. Ophthalmology 1994;101:1173-7.

17 Klein BEK, Klein R, Sponsel WE, Franke T, Cantor LB, Martone J, et al. Prevalence of glaucoma. The Beaver Dam Eye Study. Ophthalmology 1992;99:1499-504.

18 Kahn HA, Milton RC. Revised Framingham Eye Study: prevalence of glaucoma and diabetic retinopathy. $A m$ f Epidemiol 1980;111:769-76.

19 Bengtsson B. The prevalence of glaucoma. Br f Ophthalmol 1981;65:46-9.

20 Sommer A, Tielsch JM, Katz J, Quigley HA, Gottsch JD, Javitt J, et al. Relationship between intraocular pressure and Javitt J, et al. Relationship between intraocular pressure and
primary open-angle glaucoma among white and black primary open-angle glaucoma among white

21 Leske MC, Connell AMS, Wu SY, Hyman LG, Schachat $\mathrm{AP}$, the Barbados Eye Study Group. Risk factors for openangle glaucoma. Arch Ophthalmol 1995;113:918-24

22 Flanagan DW. Diabetes, glaucoma, sex and cataract (editorial). Br f Ophthalmol 1993;77:1.

23 Witkin SR, Klein R. Ophthalmologic care for persons with diabetes. $\mathscr{F} A M A$ 1984;251:2534-7.

24 Klein BEK, Moss SE, Magli YL, Klein R, Hoyer C, Johnson J. Optic disc cupping: Prevalence findings from the WESDR. Invest Ophthalmol Vis Sci 1989;30:304-9. 